DOI: $10.20472 / B M C .2018 .008 .021$

FAYYAZ AHMAD

University of Gujrat , Pakistan

\title{
AN FMRI STUDY OF NEURAL DECISION MAKING USING SUPPORT VECTOR MACHINE
}

\begin{abstract}
:
There is an increasing trend of employing fMRI by the neuroimaging community for measuring the blood oxygenation levels towards quantifying brain activity. The experimental modality typically involves a rest and a task state during which brain activation in participants is registered during an fMRI scan. In this work, we have taken this approach and analyzed two Brodmann areas (BAs), which were our regions of interest (ROI) from the prefrontal lobe of human brain, for their role in decision making. These ROIs (BA10 and BA47) are reportedly activated during decision making. Support vector machine (SVM) was employed for classification of the activated voxels in the ROls. Each dominant voxel was ranked on the basis of perceived pattern in the brain. Our experimental design and results can assist in decoding the decision-making neuron connectivity for applications in neuromarketing and neuroeconomics.
\end{abstract}

\section{Keywords:}

activated areas, decision making, fMRI, BAs, SPM

JEL Classification: D87 


\section{INTRODUCTION}

A number of different conditions have been reported as a cause for visual task for decision making: Functional magnetic resonance imaging (fMRI) and MRI techniques have been applied to visual cortices of the brain [1-3]. Particularly, the fan shapes, concentric circles and, spirals stimuli [4] have been used to detect the brain activity for example pain and visual decision.

The neural activation differences, it is commonly observed and visual stimuli provided the best information of the neurons who are related to decision making task. Cowan [5] proposed that the self-organized pattern formation on the primary visual cortex or Broadman area 17 (BA17). Artificial neural networks (ANN) models for interacting neurons can produce best patterns of activation and inactivation for the human brain [6-7] based on fMRI experiments. In the visual strait, neighboring neurons shared information for adjacent location within the visual cortex [8-10]. In the primary visual cortex of humans, a retinotopic organization has been explained with the aid of non-invasive functional MRI techniques [11-12]. Furthermore, fMRI experiments could potentially be used to test the visual perception as a result of self-organized pattern in the BA 17. An study [13] of neurons to the flickering regions can stabilize visual the hallucination in brain. It has been also confirmed that perceiving form of the visual hallucination dependent on stimuli [13].

For example, a small pattern the visual perceptions which can be helpful to understand the phenomena of decision making in the context of neuromarketing and neuroeconomics. The pattern of advertisement and marketing in the brain can be detect through reliable $\mathrm{fMRI}$ and $\mathrm{MRI}$ experiments of the consumers or population. These types of researches demonstrated phenomena that advertisement patterns recognition in human brain are governed by cognitive process, purchasing behavior and, experienced of products. Potentially, neuromarketing and neuroeconomics have been studying since 20s, but surprisingly very few experiments are exist.

The aim of our proposed fMRI experiment is to analyze the perceived patterns that are caused by two types of visual task. At the perceptual level, subject saw the easy, hard and control birds pictures. The intention of the fMRI experiment is to find out any relationship and classification among these patterns that occur at the perceptual level. 
Normally, some prefrontal areas of the brain play role for decision making particularly Brodmann areas 10 and 47 . These areas were our regions of interest (ROIs) which were reported most dominant in decision making. Some researchers [14-16] classify the cognitive process through different well-know classifiers such as logit, ANN and discriminant but our proposed model SVM provided better classification then the others.

\section{METHODOLOGY}

Support vector machines (SVMs) can recognize the pattern of brain activity as being correlated with a specific area of the brain versus another. The objective of detecting the presence of neural activations related to decision making in the brain is very complex. By Namgil and Kim [15], Support vector machines (SVMs) are pattern classification algorithms which are demonstrating high accuracy and computational efficiency in various applications. The competitiveness of SVMs is explained in two aspects [16-17]. First, SVM algorithms provide sparsely formulated hyperplane classifiers through maximization of margins [15-19]. The maximization of margins is known to be directly related to the minimization of misclassification error rates [14-19]. Second, the use of the so-called kernel functions enables the flexible non-linearization of the hyperplane classifiers [14-19]. The kernel functions measure meaningful similarities between pairs of input patterns. The SVMs become powerful by applying appropriate kernel functions to given data sets, especially [15], in case of dealing with high dimensional data sets such as fMRI data, In our two class (easy, hard) problem, a linear classifier was used as a function $f$ from a set of input vectors as $X$ ( BA 10 and BA47 activated voxels) into the set of binary labels $\{0,1\}$. SVMs take a set of training data [15] from BA17 and BA47.

$$
(x(1), y(1)), \ldots,(x(n), y(n)) \in X \times\{0,1\}
$$

and infer a classifier. In the theory of statistical learning, we assume that the values of two BAs, $(x(j), y(j)), j=1, \ldots, n$ [15] are generated from a probability distribution of BAs, $P(x, y)$. Then the goal of SVMs is to find a classifier $f$ which correctly classify the hard and easy pattern on same subject $(x, y),[15-19]$ so that $f(x)=y$.

The classifiers that SVMs choose are linear decision functions [15-19]. In case that $X$ is vector space, then activated decision functions are described as [14-19]:

$$
f(x)= \pm(<\mathcal{W}, x>+b), x \in X,
$$


where $\left\langle.,{ }_{X}\right\rangle_{X}$ represents the dot-product [14-17] in the space $X$. Choosing a linear decision function amounts to deciding the weight vector $w$ and the bias $b$. The linear decision functions can further be extended to functions that are nonlinear in $X$ by transforming.

Let $\phi: X \rightarrow H$ denote the nonlinear transformation [15-18]. Then the linear decision functions above become nonlinear as [15-19]:

$$
f(x)= \pm(<w, \phi(x)>+b), x \in X
$$

Where $w \in H$ and $b \in R$ should be estimated by a SVM.

SVMs determine $w$ and $b$ by maximizing the margin of the linear function in the space $H$. The margin is the minimal distance from input data points to the linear function [15]. Since the margin is reciprocally proportional to the square root of $\langle w, w\rangle_{H}$, SVMs attempt to minimize [15-17] $<w, w\rangle_{H}$. Among several different SVM algorithms [15-19], the 1-norm soft margin SVM is stated as follows: given a constant $C>0$, the SVM minimizes [15]

$$
\frac{1}{2}<w, w>{ }_{H}+C \sum_{i=1}^{n} \xi_{i}
$$

with respect to $w, b$, and $\xi_{i}$, subject to [15-19]

$$
\begin{gathered}
y_{j}\left(<w, \phi\left(x_{j}\right)>_{H}+b\right) \geq 1-\xi_{i}, j=1, \ldots, n, \\
\xi_{i} \geq 0, j=1, \ldots, n .
\end{gathered}
$$

Here, $\xi_{i}$ is a slack variable and it represents the margin error of $i$ th input pattern $x(j)$. By [15-19] letting $\xi_{j} \geq 0$, we are allowed to misclassify some outliers and enlarge the margin instead. To prevent the trivial case where all $\xi_{i}$ take large values, $\xi_{j}$ are penalized in 1norm sense in the above formulation.

To solve the above optimization problem, it is more convenient to deal with its dual problem [14-19]. After the Lagrange multipliers $\alpha_{i}>0$ a are introduced, the Lagrangian dual problem is derived as follows [14-19]:

$$
\begin{aligned}
& \operatorname{maximize}\left.\sum_{j=1}^{n} \alpha_{j}-\frac{1}{2} \sum_{k=1}^{n} \sum_{j=1}^{n} \alpha_{k} \alpha_{j} x_{k} y_{j}<\phi(x(j)), \phi(y(j))\right\rangle_{H} \\
& \text { subject to } 0<\alpha_{j}<C, j=1, \ldots, n
\end{aligned}
$$




$$
\sum_{j=1}^{n} \alpha_{j} y_{j}=0
$$

The optimal weight $w^{*}$ is shown to have an expansion [14-18]

$$
W^{*}=\sum_{j=1}^{n} \alpha_{j}^{*} y_{j} \phi\left(x_{j}\right)
$$

where $\alpha_{j}^{*}$ are the optimal Lagrange multipliers obtained as solutions of the dual problem. Therefore the determined SVM classifier can be written in the following form [15-19]:

$$
\left.f(x)=\operatorname{sign}\left(\sum_{j=1}^{n} \alpha_{j}^{*} y_{j}<\phi(x(j)), \phi(y(j))\right\rangle_{H}+b\right)
$$

Note that for solving the dual problem and obtaining the representation [14-19] of an SVM classifier, we don't have to specify the nonlinear transformation $\phi$, [15-19] but we only need to provide the information of the dot-products between pairs of transformed input patterns. Thus SVMs use the so-called kernel functions [15-19] to implicitly perform the dot-products. That is, for any two input patterns $x, \tilde{x} \in X$ given a nonlinear transformation $\phi$ into a dot-product space $H$, a kernel function $k$ is defined by [14-19].

$$
k\left(x_{1}, x_{2}\right)=<\phi(x), \phi(\tilde{x})>_{H}
$$

If a kernel function can be calculated efficiently, it can replace dot-product calculations (3) and (4) in SVM algorithms. In fact, every function $k$ is a valid kernel function whenever $k$ is a symmetric and positive definite real valued function and we used Gaussian kernel in our data for the accuracy of classification [15-19].

\section{DATA DESCRIPTION}

We have used functional magnetic resonance imaging study data from public site 'http://cnl.web.arizona.edu/spm.htm'. The data also presented in the estimation of parameters by a novel neighborhood approach [18]. Stimulus was presented in the easy, control and birds pictures. The Easy and hard birds consisted of 4 blocks in each conditions [16]. Time interval was two seconds between scans and there were total 80 scans of the whole brain [16]. The data were obtained using GE 1.5 Tesla [16]. The entire brain contained 17 slices and size of voxel was $64 \times 64 \times 17$. 


\subsection{DATA PRE-PROCESSING}

The MATLAB and statistical parameter mapping (SPM) were used for the analysis of fMRI human brain data. There were 3 conditions in fMRI experiment and we preprocessed data through SPM. The necessary steps of preprocess were (i) Head movement correction (ii) Spatial normalization (iii) Correction of slice timing, and (iv) Smoothing.

\subsection{ANALYSIS OF fMRI EXPERIMENT}

After preprocessing of $\mathrm{fMRI}$ study data, we estimated the model parameters of the whole brain. We have obtained SPM maps of parameters of whole brain. We also obtained SPM maps of significantly activated voxels corresponding to the hard and easy conditions. Finally, the map of activated voxels corresponding to hard verses easy condition is shown as Figure 1 in view of sagittal, Transversal and coronal slices. The activation of voxels is mostly occur in the visual and prefrontal cortices. Our regions of interest (ROI) was based on these activations of voxels which are belong to mainly Broadmann areas (BA10, BA47, BA17) by using Brain Tutor (a free software) as shown Figure 2. According to the neurological research, the neurons of BA10 and BA47 are mostly responsible for decision making in the brain.

Figure 1. SPM maps of activated voxels in the brain corresponding to hard verses easy condition in sagittal, Transversal and coronal views.

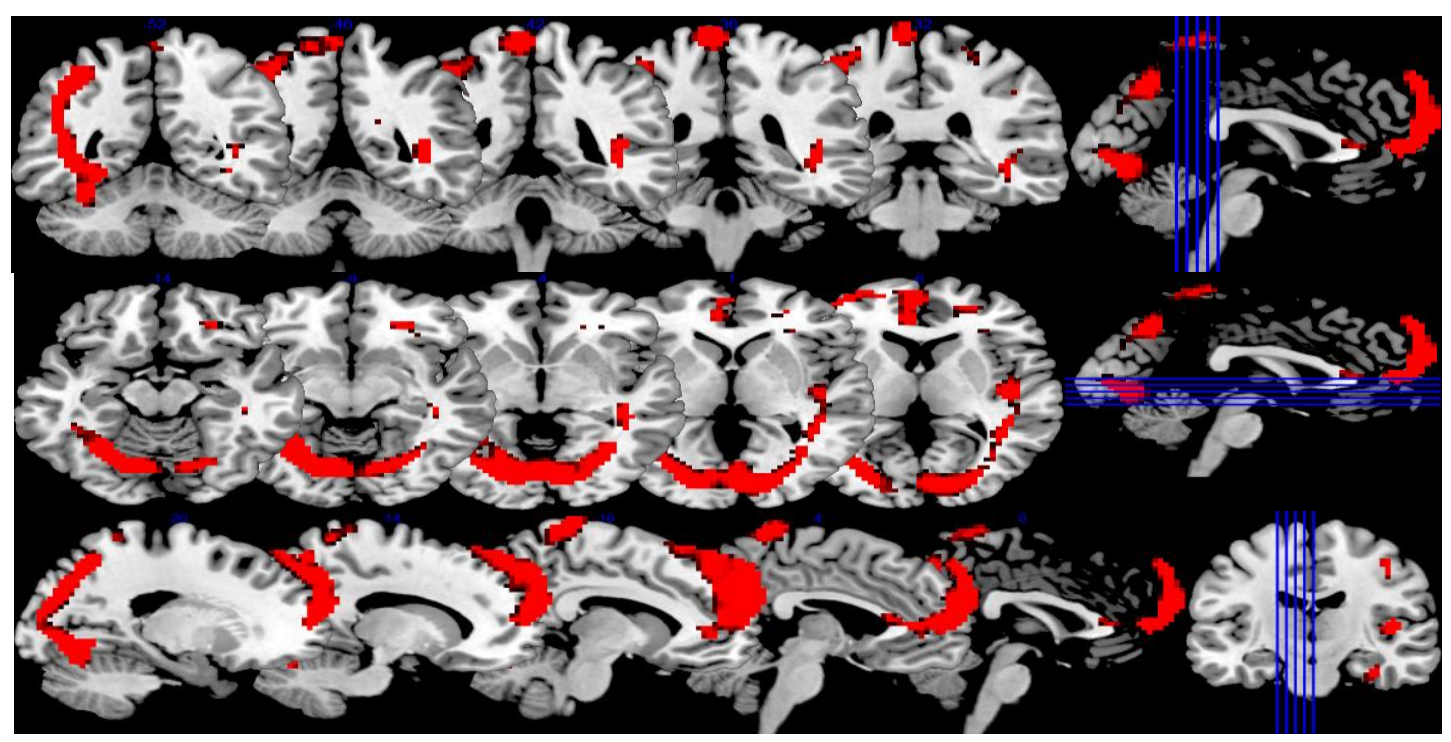


Figure 2. BA10, BA47 and BA17 areas in the views of three planes (Brain Tutor) of slices which were used for decision making

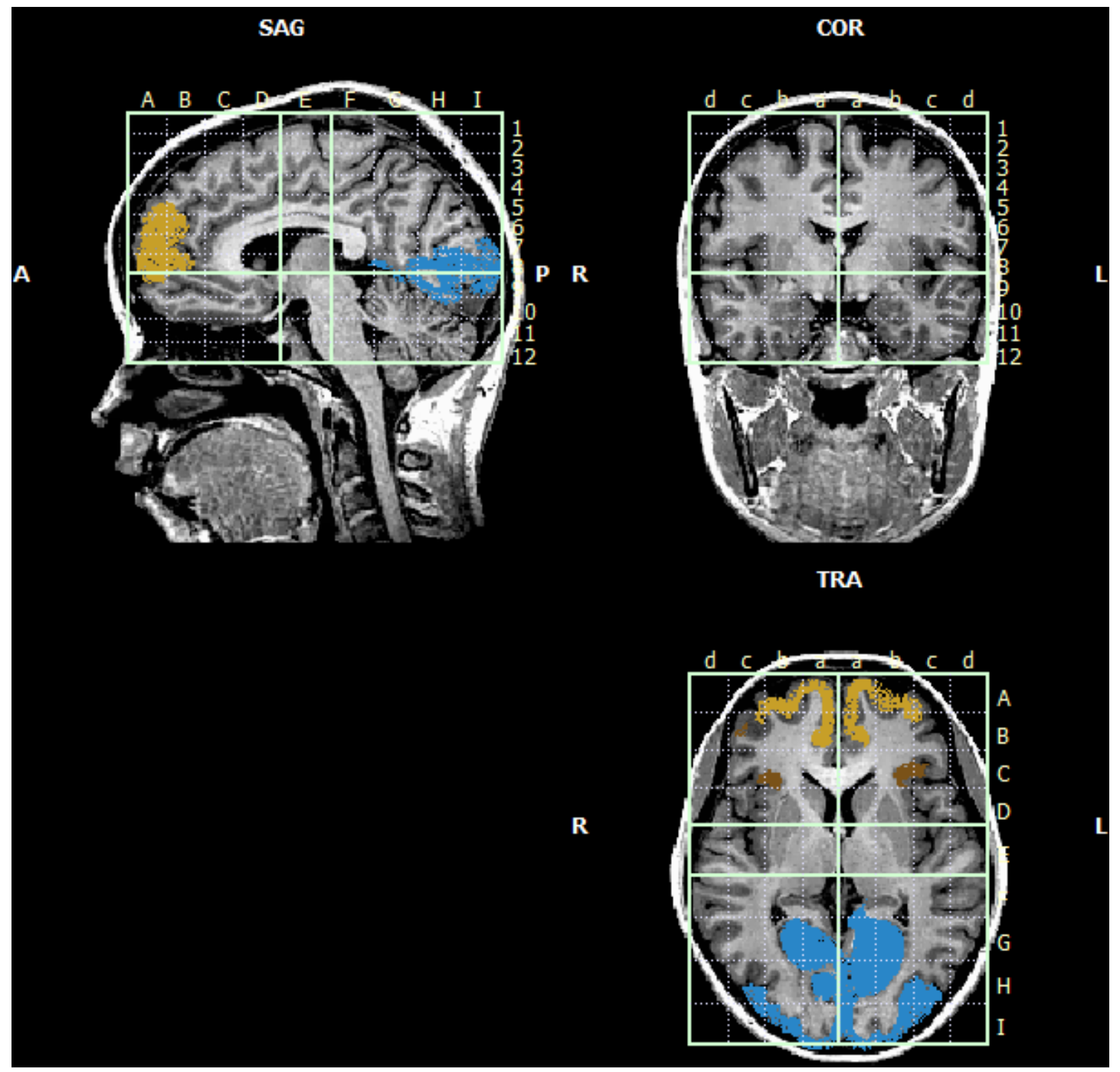

\section{RESULTS AND DISSCUSSIONS}

The other remarkable finding is that the number of significantly activated voxels of hard condition is much larger than that of the easy condition as shown in Figures 3-4. It can be further postulated that $\mathrm{fMRI}$ signals obtained by hard bird stimuli are much stronger than that of the control bird stimuli. The increased activity of the visual cortex during stimuli might explain why the visual stimuli with hard bird excits more neurons than with easy. The seeing birds stimuli might be a much real experience for subjects. The pattern of 
activation of bird's stimuli has been shown in Figure 5. Similar results were reported in terms of auditory hallucination previously. Dierks et al. [10] reported more neural activation in the temporal lobes of the brain during the perception of auditory hallucination than during auditory imagery or overt speech.

Figure 3. Activated voxels of area (BA10) corresponding to control, easy and hard birds stimuli.

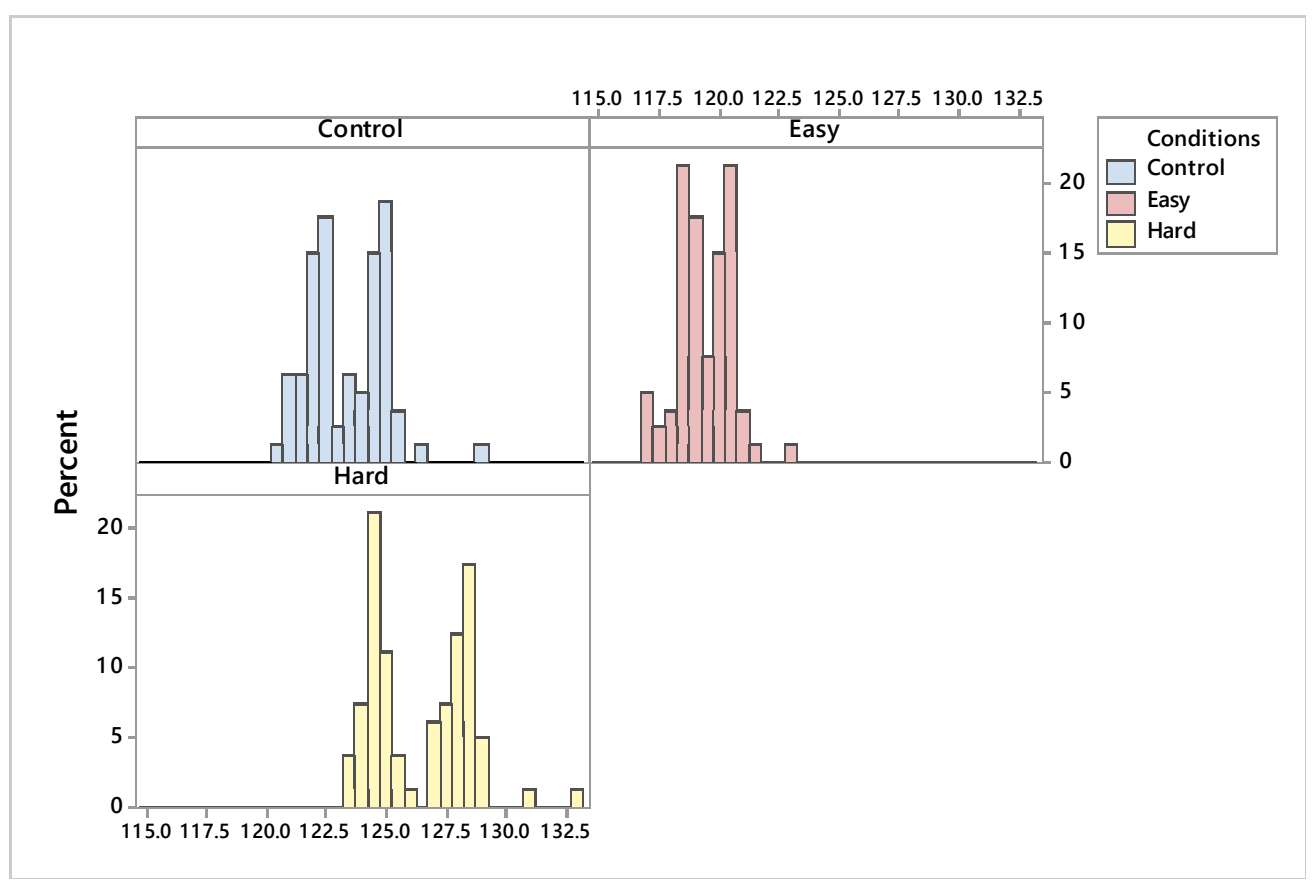

Figure 4. Activated voxels of area (BA 47) corresponding to control, easy and hard birds stimuli.

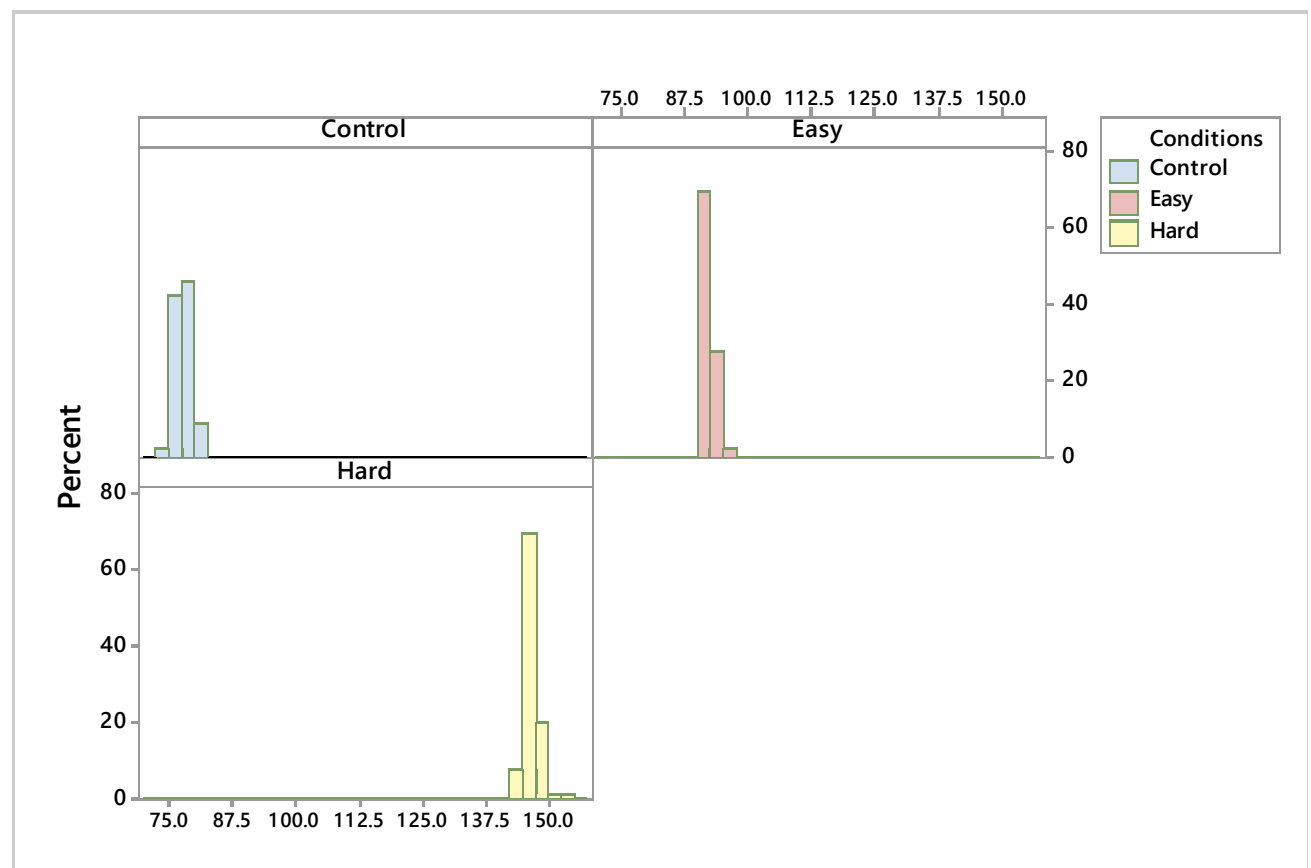


Figure 5. Pattern of activations of bird's stimuli

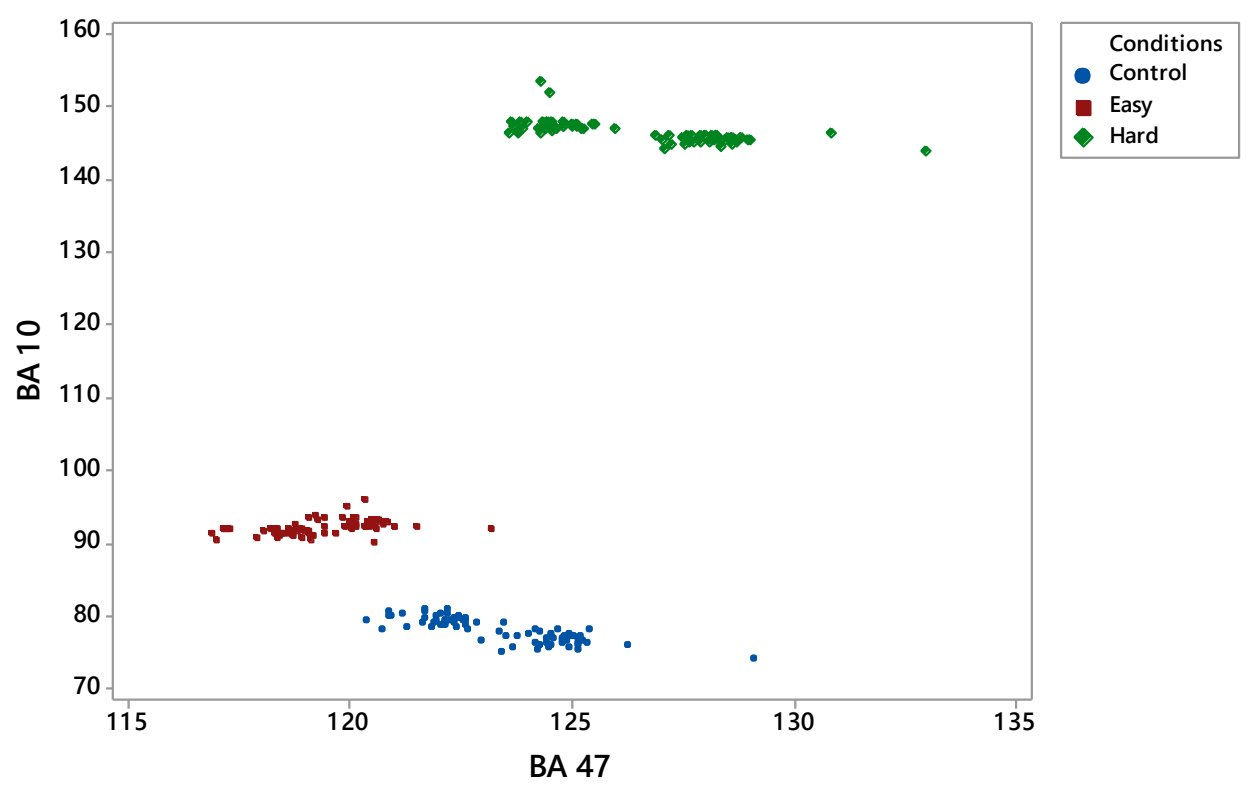

In the analysis of SVM method brain patterns of BA47 and BA10 has been shown as in Figure 5 corresponding to the bird's stimuli. According to the pattern, the classification with SVM tarined a function (3) that classify between voxel patterns of BA47 and BA10. In generalization testing: the decision making BA47 and BA10 patterns of brain activity tested with success rates of classification for the subject $93.8 \%$, and $88.6 \%$ respectively as shown in Table 1.

The receiver operator characteristics (ROC) Curve used to draw the classification rates among the binary conditions. We have obtained predicted probabilities of test sample using SVM and then we drew the ROCs (Figure 6) between easy and hard conditions to show the accuracy of our proposed model. Finally, our analysis in Table 2 showed that SVM is more reliable in decision making and has best performance as compared to other classifiers. 
Figure 6. ROC curves of SVM approach for decision making corresponding to bird's stimuli.

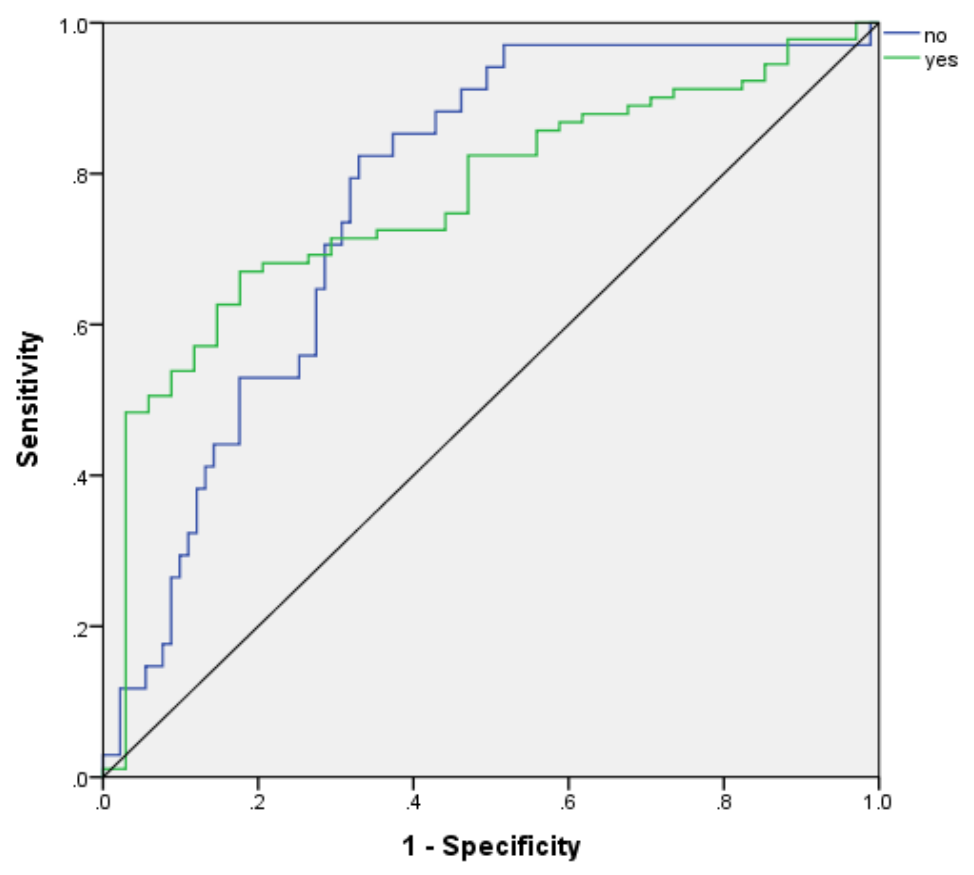

Table 1. Classification rate using SVM

\begin{tabular}{|c|c|c|c|c|}
\hline \multicolumn{4}{|c|}{ Classification } \\
\hline \multirow{3}{*}{ Sample } & \multirow{3}{*}{ Observed } & \multicolumn{3}{|c|}{ Predicted } \\
\cline { 3 - 5 } & & Easy & Hard & $\begin{array}{c}\text { Percent } \\
\text { Correct }\end{array}$ \\
\hline \multirow{3}{*}{ Testing } & Easy & 76 & 5 & $93.8 \%$ \\
\cline { 2 - 5 } & Hard & 9 & 70 & $88.6 \%$ \\
\cline { 2 - 5 } & $\begin{array}{c}\text { Overall } \\
\text { Percent }\end{array}$ & $89.4 \%$ & $93.3 \%$ & $91.3 \%$ \\
\hline
\end{tabular}


Table 2. Classification rate using different classifiers

\begin{tabular}{|l|r|}
\hline \multicolumn{2}{|c|}{ Classification rates } \\
\hline Classifiers & Rate \\
\hline ANN & $84.5 \%$ \\
\hline Logit & $67.7 \%$ \\
\hline Discriminant & $65.7 \%$ \\
\hline
\end{tabular}

\section{CONCLUSION}

We have observed that two main brodman areas are mostly activated during behavior of the consumer while making decision between products. The consumer behavior we observed through a well-known fMRI example of visual task. In fMRI experiment, they have presented easy and hard birds stimuli to the subject. We have applied SVM technique for classification between easy and hard task based on two main brodmann areas of brain. Mostly neurons were activated in those areas during the simple decision making task. We have applied different classifiers such as discriminant analysis, logistic regression, artificial neural network and SVM for classification between two types of neural activity. In our analysis, we found SVM provided best classification as compared to other classifies. SVM also is a mathematical strong approach which provided best classification rate in the context of ROC analysis. We believe that studies should help explaining mechanisms underlying this particular phenomenon. Finally, we found that SVM has better classification in the context of decision making in the human brain specially in the neuromarketing.

\section{ACKNOWLEDGMENTS}

This research supported by National Research Program for Universities (NRPU), Higher Education Commission, Pakistan, No. 7776/Punjab/NRPU/R\&D/HEC/2017.

\section{REFERENCES}

1. N. Hakjikhani, M. Sanchez del Rio, O. Wu, D. Schwartz, D. Bakker, B. Fischl, K.K. Kwong, F.M. Cutrer, B.R. Rosen, R.B.H. Tootell, A.G. Sorensen, M.A. Moskowitz, Mechanisms of migraine aura revealed by functional MRI in human visual cortex. PNAS 98 (2001) 4687-4692. 
2. R.K. Siegel, M.E. Jarvik, Drug-induced hallucinations in animals and man. In R.K. Siegel, L.J. West (Eds.), Hallucinations: Behavior, experience and theory, Wiley, New York, (1975) pp. 81 - 161.

3. W. Richard, The fortification illusions of migraine, Scientific American 224 (1971) $88-96$.

4. P.C. Bressloff, J.D. Cowan, M. Golubitsky, P.J. Thomas, M.C. Wiener, What geometric visual hallucination tell us about the visual cortex. Neural Computation 14 (2002) 473-491.

5. G.B. Ermentrout, J.D. Cowan, A mathematical theory of visual hallucination patterns. Biological Cybernetics 34 (1979) 137-150.

6. H.R. Wilson, Spikes, decisions, and actions: The dynamical foundations of neuroscience, Oxford University Press, New York, (1999) pp. $112-115$.

7. H.R. Wilson, J.D. Cowan, Excitatory and inhibitory interactions in localized populations of model neurons. Biophysical J. 12 (1972) 1-24.

8. R.D. Frostig, E.E. Lieke, D.Y. TSO, A. Grinvald, Cortical functional architecture and local coupling between neuronal activity and the microcirculation revealed by in vivo high resolution optical imaging of intrinsic signal. Proc. Natl. Sci. 87 (1990) 6082-6086.

A. Grinvald, R.D. Frostig, R.M. Siegal, E. Bartfeld, High-resolution optical imaging of functional brain architecture in the awake monkey, Proc. Natl. Sci. 87 (1991) $559-563$.

9. H.R. Wilson, F. Wilkinson, Detection of global structure in Glass Patterns: Implications for form vision, Vision Research 38 (1998) 2933 - 2947.

10. M.I. Sereno, A.M. Dale, J.B. Reppas, K.K. Kwong, J.W. Belliveau, T.J. Brady, B.R. Rosen, R.B.H. Tootell, Borders of multiple visual areas in humans revealed by functional magnetic resonance imaging, Science 268 (1995) 889 -893.

11. R.B. Tootell, J.B. Reppas, K.K. Kwong, R. Malach, R.T. Born, T.J. Brady, B.R. Rosen, J.W. Belliveau, Functional analysis of human MT and related visual cortical areas using magnetic resonance imaging, Journal of Neuroscience 15(4) (1995) 3215-30.

12. V.A. Billock, P.R. Havig, B.H. Tsou, Neural interactions between self-organized visual hallucination and physical stimuli, (2003) The manuscript submitted for publication.

13. V. Vapnik, The Nature of Statistical Learning Theory, Springer-Verlag, (1995) New York.

14. Namgil Lee Jong-Min Kim, Conversion of categorical variables into numerical variables via Bayesian network classifiers for binary classifications, Computational Statistics \& Data Analysis, 54 (2010) 1247-1265

15. James, Gareth, Witten, Daniela, Hastie, Trevor, Tibshirani, Robert. (2013). An Introduction to Statistical Learning, volume 103 XIV of: Springer Texts in Statistics.

16. Fayyaz A, Iftikhar A, Waqar M.D, Identification and using classification of voxels of human brain for rewardless-related decision making ANN technique. Neural Computing and Applications, ((2016), 2(28) 1035-1041.

17. Bey, Patrick, fMRI analysis using support vector machines (2012). 
18. Ahmad F, Ullah G. A neighborhood method for statistical analysis of fMRI data. Open Journal of Biophysics, (2012), 2(1):15-22. 